\title{
A New Approach for Operation Risk Analysis of Complex Electric System
}

\author{
Rui Chen ${ }^{1, a}$, Jie Cao ${ }^{1, b}$, Yan Wang ${ }^{1, c}$, Lingzi Zhu ${ }^{1, d}$ and Zhonghai Ma ${ }^{2, e^{*}}$ \\ ${ }^{1}$ Guizhou Power Grid Co.,Ltd, Guiyang, China \\ ${ }^{2} \mathrm{E}$-Link Information Technique Co.,Ltd, Shenzhen, China \\ achenrui@gz.csg.cn, ${ }^{b}$ caojie@gz.csg.cn, ${ }^{c}$ canoeingboy@163.com, ${ }^{\mathrm{d}} 646138067 @ q q . c o m$, \\ 55454604@qq.com
}

Keywords: Risk analysis; Failure propagation; Small-world network; Electric system

\begin{abstract}
As large-scale electric systems getting more complicated, the risk probability is also undergoing a higher tendency. This paper presents a novel risk analysis method from the complex network view. Through studying the topological structure characteristics of the electric system network, two principles are gave to assess the sensibility of the system component as network nodes. The failure diffusion intensity considering both the prior probability knowledge and the inherent safety characteristics of the system network is defined, and the failure grade diffusion process is analyzed. With the proposed method, the vulnerabilities of the system can be identified efficiently.
\end{abstract}

\section{Introduction}

The operation risk analysis for the complex electric systems is one of the mostly concerned topics today. [1]. Large-scale electric system can be considered as the set of physical elements that include a part, equipment or subsystem. The failure of a single equipment so far as to a tiny part is sufficient to cause hazards of the entire system. Traditional data-driven techniques based on the probability and statistical theory such as Bayesian network and failure modes and effects analysis (FMEA) have been widely used in many industry field. However, these methods rely on the expert's experience and knowledge greatly. In addition, they have extremely high data demand for probability assessment. As a consequence, these connatural limitations result the difficulty for identifying the potential risk when the abnormal or infrequent failures happen.

Recently, researching on the complex network theory becomes an upsurge in many field, involving from electric power transmission and communication to sociology and finance [2,3]. Many man-made network such as the WWW, the power grids and financial network exhibit similar statistical characteristics. Towards this end, a novel risk analysis method from the view of complex network theory is presented. Taking the topological characteristics of the complex electric system into account, several principles that are suit to describe the sensibility of the system network nodes are proposed. Since then, we find the failure propagation paths with high risk and corresponding critical nodes through the failure diffusion analysis. Through the simulation of failure propagation process, we can obtain the critical vulnerabilities of the system, which could be used as a support next in risk assessment and failure prevention.

\section{Small-world Properties}

The small-world network is characterized by high clustering properties yet strikingly short path lengths between pairs of nodes. These properties exist widely in many real networks, both natural and man-made domain. Watts and strogatz discover the small-world network model in 1998 which modify the regular graphs by adding shortcuts or reconnecting lines with a certain probability $p$ (see Fig.1) $[4,5]$. 


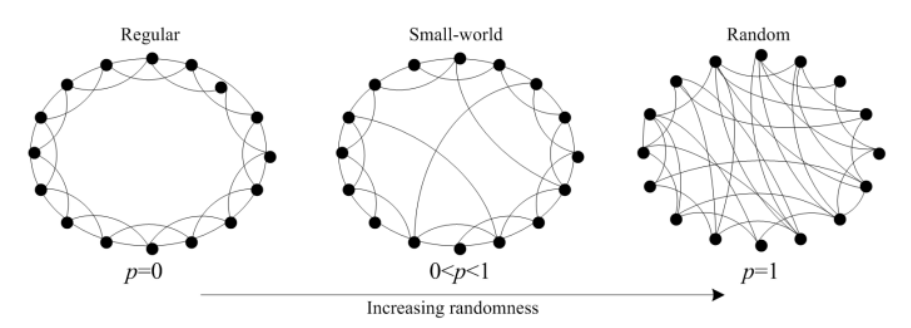

Fig.1 Small world network construction procedure

This network is described as an undirected graph $G$ which has $N$ nodes and $K$ edges. The graph can be described by an $N \times N$ symmetric connection matrix $E=\left\{E_{i j}\right\}, i=1 \ldots N, j=1 \ldots N$, where $e_{i j}$ is 0 if there is no edge between node $i$ and node $j, 1$ for the opposite case. Topologically, the small-world network can be described with several statistical coefficients as follows:

The average value of node degree: Supposing the degree $k_{i}$ of a node $i$ is defined as the number of edges rooted in it. The average value of node degree $K$ over the network is defined as:

$$
K=\frac{1}{N} \sum_{i} k_{i}, \quad i=1, \ldots, N .
$$

The characteristic path length: The characteristic path length $L$ is defined as the average over the network of the shortest path length between any two nodes. It is defined as:

$$
L=\frac{1}{N(N-1) / 2} \sum_{1 \leq i, j \leq N} D(i, j)
$$

where $D(i, j)$ is the shortest path length between two nodes $i$ and $j$. From the definition $D(i, j)$ is greater or equal than 1 , being equal to 1 if there exists a direct connection between $i$ and $j$.

The clustering coefficient $C$ that measures the average probability for tow nodes, having a common neighbor, to be themselves connected. It is gave by:

$$
C=\frac{1}{N} \sum_{i=1}^{N} \frac{2 t_{i}}{k_{i}\left(k_{i}-1\right)}
$$

In which $t_{i}$ is number of edges existed between the neighbors of node $i$. If a network has the small-world phenomenon, it often satisfies the statistic characteristic as follows:

$$
\left.\begin{array}{c}
C>C_{r} \\
L \geq L_{r}
\end{array}\right\}
$$

where $C_{r}$ and $L_{r}$ are the clustering coefficient and characteristic path length of the random network respectively, and $C_{r} \sim K / N, L_{r} \sim \ln (N) / \ln (K)$.

Above parameters are used to formalize the characteristics of the small world phenomena. The small world network can be described with a high clustering coefficient (typical of regular networks) and short characteristic path lengths (typical of random networks). The small world phenomena also exist in the complex electric system network and these coefficients are useful for the operation risk analysis.

\section{The Complex Network Method to Risk Analysis}

Framework of Complex Electric System. To obtain a reasonable purview, a complex electric system can be naturally described as a logic network. This network is composed of many interacting entities (electrical equipments and devices) with anfractuous interaction relationship. Each component of the system is networked with others by the connection of the hardware linkages (i.e. the electric wire, communication cables). By decomposing the system structure, we abstract the logic nodes from the electric system topology structure. In this way, a simplified network model is established to describe the architecture of the complex electric system. Since then, we utilize an $N \times N$ interaction matrix $C$ to store the potential relationship among the nodes. Through the interaction matrix, we can achieve the key topology parameters such as the degree of the each network node, the characteristic path length and the clustering coefficient of the electric system network. Building and constructing system network model 
delays on the experience of the analyzers, and it is a subjective process.

Critical Nodes of Electric System. In most case, the topological network implicates some important features of the electric system. By researching the electric system network, we discover that the small-world properties will accelerate the failure propagate rapidly within the whole system. Generally, the clustering coefficient influences the failure diffusion extent and the characteristic path length influences the depth. Owing to the high clustering and strikingly short path lengths, both the failure diffusion speed and propagation scope within the small-world network are extremely higher than the regular or random networks.

Mostly, the node's degree and the number of the shortest paths are the important factors in electric system failure. The larger degree of the nodes is, meaning the more failure diffusion paths will be. In addition, we define the total number of shortest paths passing through the node $v$ as the node's load $l(v)$ [6]. Supposing the failure diffusion is often along the shortest paths, the breakdown of the nodes with large load will cause interactive influence on other nodes fast. As a result, we arrive at the following general rules: (a) big degree of node means wide diffusion scope, (b) high load of node indicates rapid propagation speed.

By focusing on the researching of small world phenomenon, we can reveal several useful principles for assessing the safety characteristic of the complex electric system. The nodes with big degree and/or high load can be considered as the critical nodes of the complex electric system which need be protected and maintained especially.

\section{Failure Diffusion Analysis}

When using the small-world properties to the operation risk analysis for complex electric system, we would like to apply the topology statistics to find the critical nodes which play an important role in maintaining stability of the entire system. In the study of any failure, a broad spectrum of failure modes should be considered. When the cascaded failure happens, the failure component will cause the neighbors to fail immediately or promote the deterioration of the affected ones step-by-step. In practice, it is impossible and unnecessary to find every failure modes of each component simultaneously. One possible method for risk analysis is to simulate the dynamic process of the failure diffusion with an abstract pattern, which means the different external modes of the system failures can be ignored to some extent.

Therefore, we give a simple model for representing failure diffusion process within complicated electric system networks. Initially the network is in a stable state that means all the nodes are operating well. Then, as the failure or damage of a node happening, the adjacent nodes will be influenced through the interactive manner. As a result, the entire network will be disturbed in a short time. That means the interactive influence will lead all the affected nodes to fall into failure gradually. Eventually the cascaded failure will result in the disabled state or the performance reduction of the whole system. This diffusion process is similar to the cascading failures appearing in complex electrical system (see Fig.2).

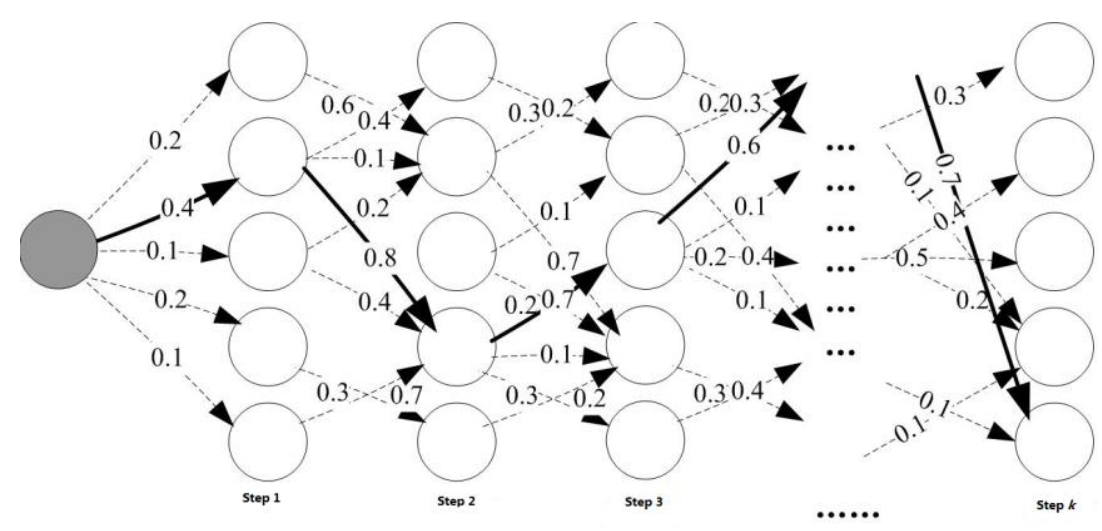

Fig. 2 Failure diffusion process 
Traditional risk analysis techniques apply the probability approach to estimate the possible propagation direction of the certain failure. However, it is impossible to gain the accurate probability data in practice. In order to solve this problem, we define the failure diffusion intensity $I$ instead of the propagation probability and assign it to each edge as a weight. As aforementioned, the load at a given node $v, l(v)$, is defined as the number of shortest paths between pairs of nodes crossing it. Analogously we define the load at the edges $e, l(e)$, as the number of shortest paths crossing a given edge. More loads an edge has, more nodes would fall into failure fast by the failure propagation via this edge. Consistently with that assumption, we formulate the failure diffusion intensity $I_{i j}$ of an edge $e_{i j}$ starting from $i$, going to $j$, as:

$$
I_{i j}=w_{p} p\left(e_{i j}\right)+w_{s} l\left(e_{i j}\right) / \sum_{i \neq j} l\left(e_{i j}\right) \quad 1 \leq i, j \leq n
$$

where $p\left(e_{i j}\right)$ and $l\left(e_{i j}\right)$ are the prior failure probability and the load of the edge $e_{i j}$. In addition, $w_{p}$ and $w_{s}$ are the weight for the prior probability and the load. In this way, we can define he failure diffusion intensity considering both the prior probability knowledge and the inherent safety features of the complex system topology.

\section{Conclusion}

This paper presents a prospective research of the electric system risk issues from a new viewpoint of the complex network theory. By focusing on the general characteristics of interactive influence among the different entities of the complex electric systems, we give some useful principles for identifying system vulnerabilities. Then, we provide precise descriptions for the failure diffusion process, so that the major results can be concluded. Our study reveals that those nodes or edges with high load are more safety sensitive than the others, and the safety performance of a complicated system with small-world properties is really influenced by the availability of these critical nodes and edges.

Though the present method is deterministic in nature, but it should take the uncertain factors into account. Thus, how to provide the uncertainty analysis ability by extending the current model with the probabilistic theory should be study in the future.

\section{References}

[1] T.X. Zheng, E. Litvinov: Power and Energy Society General Meeting (Detroit, MI, USA, December 15, 2011). Vol.19, p.1.

[2] M.E.J. Newman: SIAM REVIEW, Vol.45 (2003) No.2, p.167.

[3] X.F. Wang, G. Chen: IEEE Circuits and System Magazine, Vol.3 (2003) No.1, p.6.

[4] D. J. Watts, S. H. Strongatz: Nature, Vol.393(1998) No.4, p.440.

[5] D.J. Watts: Annual Review of Sociology, Vol.30 (2004), p.243.

[6] A. E. Motter, Y. C. Lai: Phys. Rev. E, Vol. 66 (2002) No.62, p. 065102. 